\title{
Involvement of Slit-Robo signaling in the development of the posterior commissure and concomitant swimming behavior in Xenopus laevis
}

Yasuhiko Tosa ${ }^{1 \dagger}$, Kiyohito Tsukano ${ }^{1 \dagger}$, Tatsuya Itoyama', Mai Fukagawa', Yukako Niii, Ryota Ishikawa', Ken-ichi T. Suzuki ${ }^{3}$, Makiko Fukui ${ }^{1}$, Masahumi Kawaguchi ${ }^{2}$ and Yasunori Murakami ${ }^{1 *}$

\begin{abstract}
Introduction: During vertebrate development, the central nervous system (CNS) has stereotyped neuronal tracts (scaffolds) that include longitudinal and commissural axonal bundles, such as the medial longitudinal fascicle or the posterior commissure (PC). As these early tracts appear to guide later-developing neurons, they are thought to provide the basic framework of vertebrate neuronal circuitry. The proper construction of these neuronal circuits is thought to be a crucial step for eliciting coordinated behaviors, as these circuits transmit sensory information to the integrative center, which produces motor commands for the effective apparatus. However, the developmental plan underlying some commissures and the evolutionary transitions they have undergone remain to be elucidated. Little is known about the role of axon guidance molecules in the elicitation of early-hatched larval behavior as well.
\end{abstract}

Results: Here, we report the developmentally regulated expression pattern of axon-guidance molecules Slit2 ligand and Robo2 receptor in Xenopus laevis and show that treatment of X. laevis larvae with a slit2- or robo2-morpholino resulted in abnormal swimming behavior. We also observed an abnormal morphology of the $P C$, which is part of the early axonal scaffold.

Conclusion: Our present findings suggest that expression patterns of Slit2 and Robo2 are conserved in tetrapods, and that their signaling contributes to the construction of the PC in Xenopus. Given that the PC also includes several types of neurons stemming from various parts of the CNS, it may represent a candidate prerequisite neuronal tract in the construction of subsequent complex neuronal circuits that trigger coordinated behavior.

\section{Introduction}

External stimuli received by several types of sensory receptors located on the body's surface are transferred to the peripheral nerves. Subsequently, the nerve afferents enter the brain and send information to relay nuclei, which in turn project to higher centers in which the various sensory inputs are integrated. The motor center then outputs commands to motoneurons located in the hindbrain or in the spinal cord. Thus, the construction of a precise circuit is a crucial step in eliciting appropriate behavioral responses. If such neuronal circuits are disorganized during

\footnotetext{
* Correspondence: murakami.yasunori.mu@ehime-u.ac.jp

${ }^{\dagger}$ Equal contributors

${ }^{1}$ Graduate School of Science and Engineering, Ehime University, 2-5

Bunkyo-cho, Matsuyama 790-8577, Japan

Full list of author information is available at the end of the article
}

ontogenesis, early larvae may be unable to perform coordinated body movements. In the developing vertebrate central nervous system (CNS), early-differentiating neurons extend axons toward their target regions, forming stereotyped tracts (scaffolds) consisting of longitudinal and commissural axonal bundles [1-8]. In later development, these early tracts are thought to serve as guideposts for later-developing axons [9]. The basic framework of these tracts is highly conserved in vertebrate evolution $[2,10]$. The early tracts consist of longitudinal (extending along the anteroposterior axis) and commissural (connecting to the left and right side of the brain) tracts. The former include the lateral longitudinal fascicle, tracts of the postoptic commissure (TPOC), and the supraoptic tract (SOT); the latter include the anterior, habenular $(\mathrm{HC})$, and posterior $(\mathrm{PC})$ commissures. 
Developing vertebrate brains are typically subdivided into series of segments called neuromeres, and those located in the diencephalon are called prosomeres [11-17]. It is known that some commissural bundles are located at a specific region on the neural tube corresponding to prosomeric compartments. The HCs and PCs are formed in prosomere 2 (thalamus) and prosomere 1 (pretectum), respectively, in many vertebrate groups [14]. The highly conserved framework of these commissures implies that a strictly maintained neurodevelopmental program involved in their wiring has been inherited during vertebrate evolution. In fact, some transcription factors and axonguidance molecules have been shown play an important role in the formation of the network of these early tracts [18-20]. Previous studies have revealed that the interaction between Slit (ligand) and Robo (receptor), which acts as a repulsive guidance signal, plays an essential role in the formation of early scaffolds, e.g., the inhibition of Slit2 or Robo2 results in an abnormal morphology of the TPOC [21-23] and SOT [24]. These molecules are also involved in the formation of commissural tracts in insects [25, 26] and vertebrates (Slit:[27, 28]; Robo:[29, 30]). In zebrafish robo3 mutant, the axons of the Mauthner neuron fail to cross the midline [31]. In mammals, the Slit/Robo interaction is involved in the formation of the corpus callosum, which is a type of commissural system that connects the cerebral hemispheres [32]. The similarity of the Slit-Robo interaction in teleosts and rodents leads to the possibility that the role of slit and robo is conserved in vertebrate evolution. To study such evolutionary conservation in the vertebrate lineage, it is important to study the function of slit and robo in amphibians, as these animals are thought to have diverged between the teleost and amniote lineages. In addition, the development of locomotion patterns in larval stages is well-described in anuran species [33-35]. For these reasons, Xenopus laevis, an anuran species, may be a suitable model for use in phylogenetic studies and behavioral analysis in early hatched larvae. The aim of the present study is to identify the role of Slit-Robo signaling in the formation of early tracts and/or the elicitation of swimming behavior in Xenopus. We also tried to identify the evolutionary transition of Slit-Robo signaling. To this end, we studied the expression pattern of Slit2 and Robo2 in Xenopus embryos, then perturbed their signals using morpholino antisense oligonucleotides (MO) and analyzed the swimming behavior of early larvae. We found that expression domains of Slit2 and Robo2 in Xenopus are similar to those of amniotes, indicating that the axon guidance mechanism that depends on Slit-Robo signaling is evolutionarily conserved in the forebrain of tetrapods. We also found a disorganized swimming behavior and an abnormal morphology of the PC in both slit2- and robo2-MOinjected larvae. These results indicate that interaction between Slit2 and Robo2 is involved in the construction of the PC and the formation of neuronal element(s) that control coordinated body movement in Xenopus larvae.

\section{Materials and methods Xenopus embryos}

Adult Xenopus laevis were purchased from a local farm (Hamamatsu Seibutsu Kyozai Co. Ltd; Shizuoka Prefecture, Japan), and fertilized eggs were obtained in the laboratory via artificial fertilization. Fertilized eggs were then placed in fresh water and incubated at $20{ }^{\circ} \mathrm{C}$. Embryonic stages were determined based on Nieuwkoop and Faber (1967) [36]. The studies were performed according to the Ethical Guidelines for Animal Use of the Animal Care Committee at Ehime University.

\section{Isolation of axon-guidance genes in X. laevis}

Xenopus homologues of slit 2 and robo 2 were isolated by polymerase chain reaction (PCR) using $X$. laevis embryonic cDNA as a template. The primers of Xlslit2 were designed based on a published sequence (NM_001087668.1). The following primers were used: Xlslit2-F, 5'-TGAATC AGCACCACCAATGG-3, Xlslit2-R, 5'-CTAGTCTCG ATACCTTCTCG-3'; Xlrobo2-F, 5'-TGGATTGTAGAG TGCTGAGG-3', Xlrobo2-R, 5'-CACGGAGCAATGCT ACTTCC-3'.

The PCR products included in the agarose gel were purified using the Wizard SV Gel and PCR Clean-Up System (Promega), and the DNA fragment was cloned into pGEM-T Easy (pGEM-T Easy Vector Systems; Promega).

\section{Injection of morpholinos}

To inhibit Slit2 or Robo2 signals specifically in neuronal tissues, a slit2 MO (GCCACCCAAGGAAAGAACCC AACCA; Gene Tools, LLC) and a robo2 MO (AGCCA CCAGAAAGCCCATGTTTCCC) were injected at the 8-cell stage into the small blastomere of the animal pole, which differentiates into the CNS [37]. To visualize injected cells, enhanced green fluorescent protein (eGFP) mRNA was co-injected into the blastomere. Morpholinos containing five mismatched nucleotides were used as a control. As controls of slit2 and robo2, GCgAgCCAAcG AAAcAAgCCAACCA and AGCCACgAcAAAcCCgAT cTTTCCC were used, respectively (the mismatched nucleotide is shown in lower case). We injected $2.5 \mathrm{pmol}$ of slit2, robo2, and control MOs. Injected embryos were incubated in $3 \%$ ficoll until the blastula stage; subsequently, embryos were replaced in $0.3 \times \mathrm{MMR}(100 \mathrm{mM} \mathrm{NaCl}$, $5 \mathrm{mM} \mathrm{MgCl}_{2}, 0.5 \mathrm{mM} \mathrm{CaCl}$, $5 \mathrm{mM}$ EGTA, $20 \mathrm{mM}$ HEPES-NaOH, pH 7.5) at $20{ }^{\circ} \mathrm{C}$. At stage 28 , we checked eGFP illumination under a fluorescence microscope (Lumar V12; Carl Zeiss SMT GmbH, Oberkochen, Germany). Embryos showing neuron-specific localization of eGFP were incubated and used for further analyses. 


\section{Morphological observation}

Paraformaldehyde (PFA)-fixed specimens were observed under a stereomicroscope (Lumar V12; Carl Zeiss). The body length, curvature of the body axis, and surface area of the eyes were measured using the Axio Vision software (release 4.7.2; Carl Zeiss). Ten larvae were examined in each measurement.

\section{Behavioral analysis}

Behavioral analyses at the early larval stage (stage 46-47) were performed as described previously [38], with minor modifications (Fig. 1). A hatched larva was transferred to a small aquarium $(130 \times 150 \mathrm{~mm})$ on a light board, and its swimming behavior was recorded for 3,000 frames (0.03 $\mathrm{s} /$ frame) using a video camera placed above the dish (Himawari GE60; Library Co. Ltd, Tokyo, Japan). Fifteen organisms were examined per experiment. The swimming trajectory was visualized on a personal computer, and the swimming speed and distance were measured using the Move-tr/2D7.0 software (Library Co. Ltd). To calculate the swimming pattern, the maximum and the minimum values of the swimming trajectory in

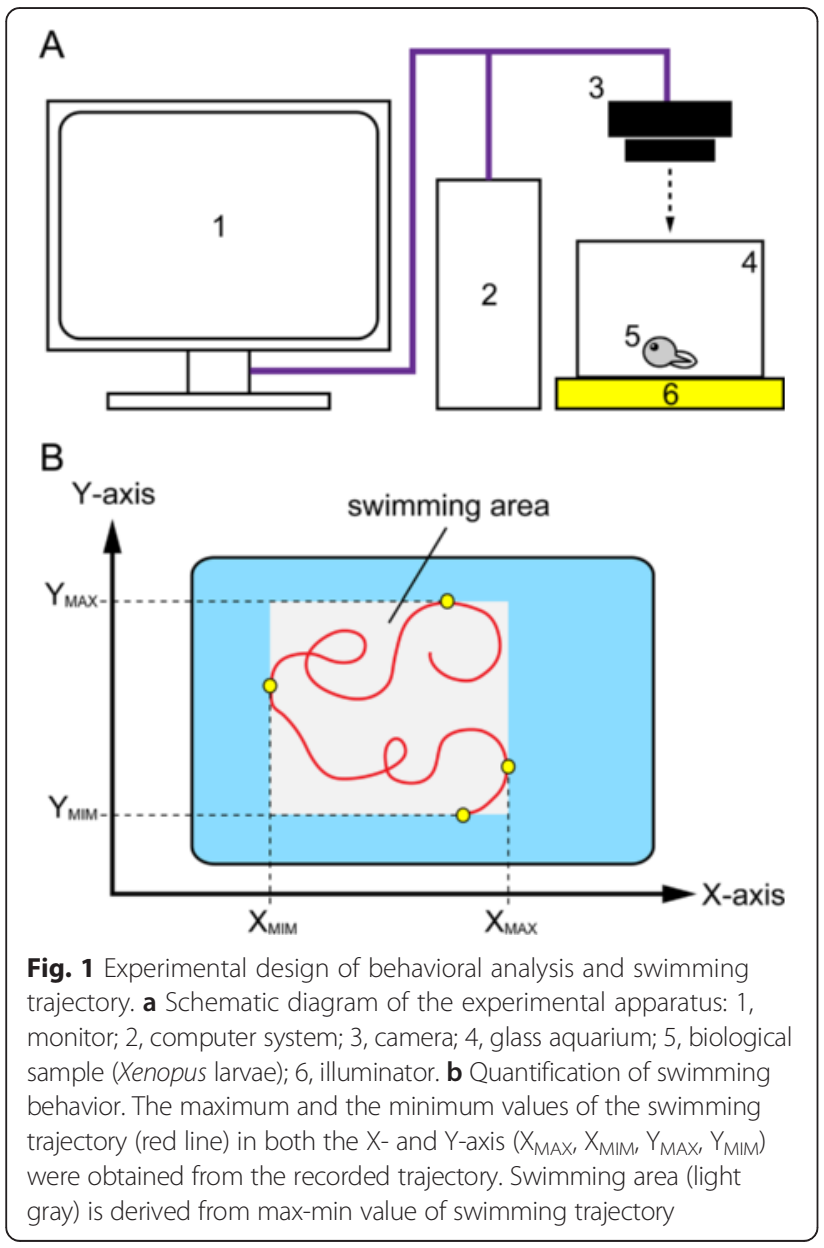

both the $\mathrm{x}$ - and the $\mathrm{y}$-axis were obtained as the swimming area (see also [38]). The whole experiment was performed twice. Significant differences in each variable among the subunit types were examined by Scheffé test after one-way analysis of variance (ANOVA) to characterize each subunit type.

\section{Axonal labeling of the posterior commissure}

For retrograde labeling of axons using NeuroVue, Xenopus larvae were dissected in phosphate-buffered saline (PBS, pH 7.5). After fixation in $4 \%$ PFA in PBS, a small chip of NeuroVue Red (Funakoshi 24835) was inserted into the primordium of the pretectum. Larvae were then incubated at $37^{\circ} \mathrm{C}$ for one week in $2 \%$ PFA in PBS. Labeled specimens were dissected, and the isolated brain was sectioned at $50 \mu \mathrm{m}$ using a vibratome (EM PRO7; Dosaka EM Co. Ltd, Kyoto, Japan) after embedding in $5 \%$ agar. Sections were then observed under a confocal microscope (LMS 510 META; Carl Zeiss).

\section{Whole-mount in situ hybridization}

For whole-mount in situ hybridization, after fixation in $4 \%$ PFA, embryos were dehydrated in a graded series of methanol (30\%, $50 \%, 70 \%, 90 \%$, and $100 \%)$ and stored at $-25{ }^{\circ} \mathrm{C}$. In situ hybridization was performed as described previously [39], with minor modifications. After the color development, specimens were dissected, and the isolated brain was observed under a stereomicroscope (Carl Zeiss). Some specimens were cut into $50 \mu \mathrm{m}$ using a vibratome after embedding in $5 \%$ agar, and were then observed under a microscope (Axio Image A1; Carl Zeiss).

\section{Whole-mount immunohistochemistry}

As a primary antibody to visualize developing axons, we used a monoclonal antibody raised against acetylated tubulin (T-6793, diluted 1/1000; Sigma-Aldrich). Developing muscles were stained with MF20, which recognizes myofilaments (obtained from the Developmental Studies Hybridoma Bank, University of Iowa; diluted 1/ 100). As a secondary antibody, we used Alexa Fluor 555 goat anti-mouse IgG $(\mathrm{H}+\mathrm{L}$; A21422; Invitrogen $)$ or Alexa Fluor 488 goat anti-mouse IgG $(\mathrm{H}+\mathrm{L}$; A11001; Invitrogen). Nucleus was labeled with DAPI (D9564, $1 \mathrm{mg} / \mathrm{mL}$, Sigma-Aldrich). For whole-mount immunostaining experiments, hatched larvae were prepared as described previously $[38,40]$. The stained specimens were observed under a fluorescence microscope (Lumar V12; Carl Zeiss) or a confocal microscope (510 META; Carl Zeiss). Ten larvae were examined in each measurement. Statistical analyses were carried out using Student's $t$-test. 


\section{In situ hybridization combined with} immunohistochemistry

Whole-mount in situ hybridization was performed as described above. Then, the samples were washed several times with Tris-buffered saline with $0.1 \%$ triton-x 100 (TBST). The nerve fibers were visualized by an immunohistochemistry by described above. Briefly, samples were incubated for 1 overnight in TBST containing $5 \%$ skim milk (TSTM). They were then treated with the antiacetylated tubulin antibody. Samples were incubated in the antibody for 2 days at RT in TSTM containing $0.02 \%$ $\mathrm{NaN}_{3}$. The samples were then washed in TBST four times and subsequently incubated in the secondary antibody (Alexa488 anti-mouse IgG, diluted 1:500) for 2 days at RT in TSTM. The samples were then washed four times in TBST. The specimens were observed under a fluorescence stereomicroscope (Lumar V12; Carl Zeiss).

\section{Results}

\section{Swimming behavior in MO-treated larvae}

To study the molecular functions of Xenopus cognates of slit2 (Xlslit2) and robo2 (Xlrobo2) in the developing nervous system, $\mathrm{MO}$ were injected into the blastomere, which differentiates into the CNS. Although many MO-injected individuals showed no abnormal morphology at stage 44, a small number of larvae exhibited head curvature or eye reduction in both the control- and slit2-MO-injected groups. Conversely, the robo2-MO-injected specimens and their control-MO-injected specimens showed a more asymmetrical craniofacial morphology than did slit2-MOand control-MO-injected specimens. Thus, in the subsequent analysis, we used slit2-MO-injected larvae and their control-MO-injected larvae (with normal body morphology), whereas we used robo2-MO-injected larvae and their control-MO-injected larvae, which have a slightly asymmetrical shape in the head or eye, in addition to larvae with normal body morphology.

As Slit2 or Robo2 is involved in the formation of the early neuronal circuit in many vertebrates [27-30], we hypothesized that MO-treated larvae possess abnormalities not only in their neuronal elements, but in behavior as well. In fact, it has been reported that early-born neuronal frameworks play an essential role in the regulation of body movement [41]. Previous studies have shown that the first alternating body movements in $X$. laevis occur on both sides of the body during the early swimming stage (stages 28-33); however, the embryo does not move through the water. The myotomal musculature is fully developed during the free-swimming stage (from stage 33), and the embryo is able to move through the water and swim [33-35]. Larvae begin swimming to search for food at the early larval stage (from stage 45 on), at which point their oral apparatus becomes functional, enabling them to eat. As this free swimming is thought to be an initial behavior in this animal that is controlled mainly by early-born neuronal circuits, without modification by the postnatal experience, this swimming behavior is expected to be useful for the study of the function of neuronal networks constructed by an intrinsic genetic program. To test whether larvae treated with Xlslit2 or Xlrobo2 MO perform the correct movement, we analyzed the swimming pattern of larvae (stage 46-47) in which active swimming was continuously observed. Our behavioral analysis revealed that specimens that were treated with control MO swam in a large circular trajectory in a coordinated manner (Fig. 2b). In contrast, larvae treated with Xlslit2 $\mathrm{MO}$ exhibited an abnormal swimming pattern of very small circles with unusual movements (Fig. 2c). A similar phenotype was observed in Xlrobo2-MO-treated larvae (Fig. 2i). The swimming areas traversed by larvae treated with Xlslit2 and Xlrobo2 MO were significantly smaller than those of control animals (Fig. 2d, j). In addition, the overall swimming distance and speed were also decreased significantly in Xlslit2- and Xlrobo2-MO-injected larvae compared with the controls (Fig. 2e, f, k, l).

\section{External morphology and musculature construction}

We next studied the morphology of the larvae used in the behavioral study to determine whether these larvae exhibited any morphological defects. Initially, we observed the trunk musculature and peripheral nerves, both of which are thought to be important for coordinated swimming. Immunostaining with an anti-myofilament antibody revealed that the segmented myomeres were arranged normally in the trunk region of Xlslit2- or Xlrobo2-MO-treated larvae, as well as in controls (Fig. $3 \mathrm{a}-\mathrm{c})$. In addition to the muscular system, Xlslit2- or Xlrobo2-MO-treated larvae exhibited a normal innervation pattern of the spinal nerves in the dorsal part of the trunk (arrowheads in Fig. 3d-g). High magnification images showed the segmentally innervating spinal nerves between myomeres (Fig. 3h-k). Furthermore, Xlslit2- or Xlrobo2-MO-treated larvae represented apparently normal morphology of the craniofacial peripheral nerves compared to those of control specimen (Fig. 31-o), and we did not observe abnormal morphology of the cranial and the optic nerves in MO-treated larvae (Fig. 3p-s). Overall, there were no severe morphological problems in the musculature and peripheral nerves in the larvae used in the behavioral study, although, as noted above, some Xlrobo2and its control-MO-injected specimens showed curvature of the brain and eye reduction (data not shown).

\section{Expression of XIslit2 in Xenopus larvae}

To identify the function of XISlit2 and XIRobo2 on the process of formation of the neuronal circuit, we studied the expression patterns of transcripts encoding those 
A

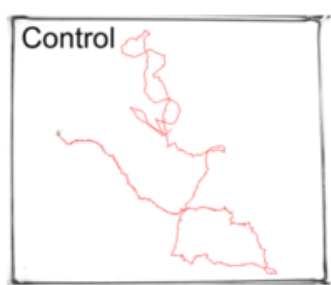

D

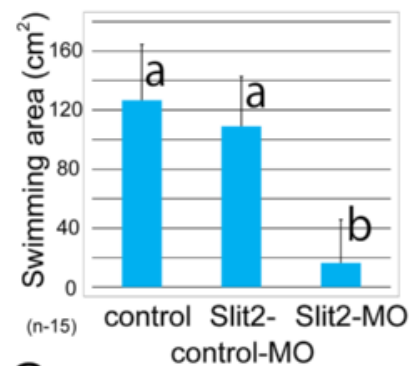

G

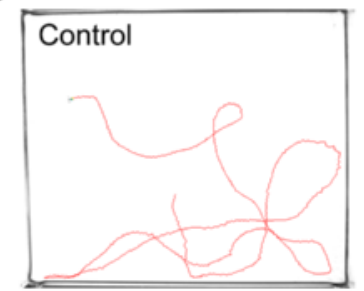

$\mathrm{J}$

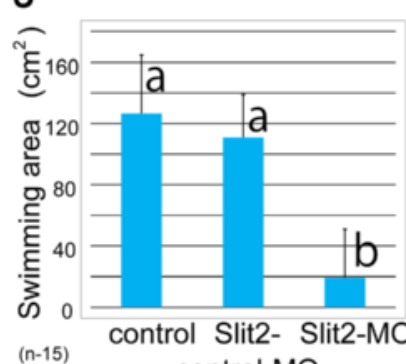

B

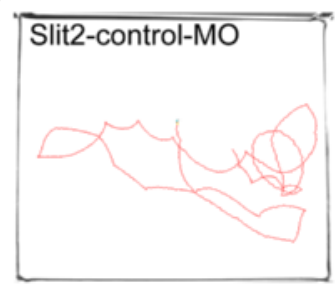

E

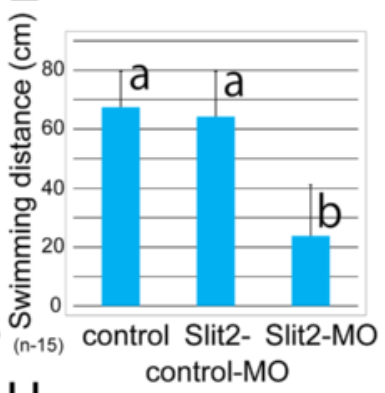

$\mathrm{H}$

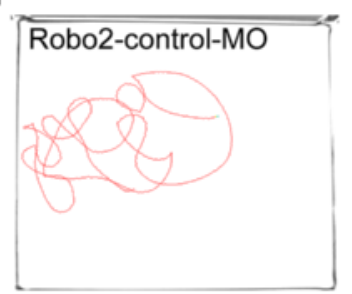

$\mathrm{K}$

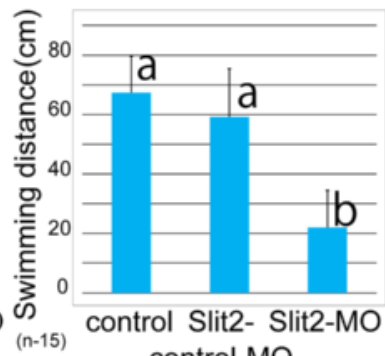

C

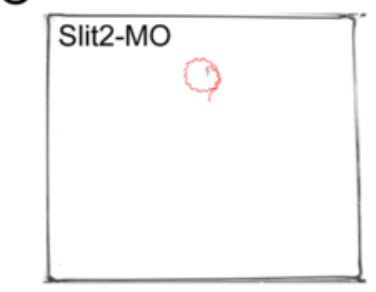

$\mathrm{F}$

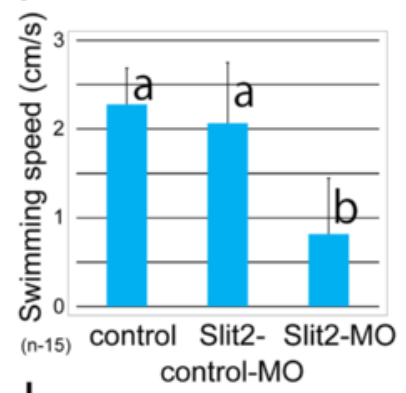

|
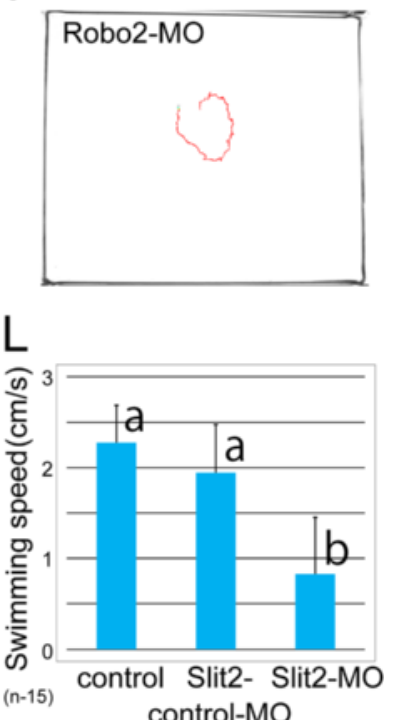

Fig. 2 slit2- or robo2-MO-injected tadpoles indicates an abnormality in swimming behavior. $\mathbf{a}-\mathbf{c}, \mathbf{g}-\mathbf{i}$ Red lines show the swimming trajectory of tadpoles: a, g Un-injected control, b Slit2-control-MO, c Slit2-MO, h Robo2-control-MO, i Robo2-MO. d-f, j-I Quantification of the swimming area $(\mathbf{d}, \mathbf{j})$, swimming distance $(\mathbf{e}, \mathbf{k})$ and swimming speed $(\mathbf{f}, \mathbf{I})$. In the slit2- or robo2 MO-injected larvae, values of all items measured are significantly decreased. $\mathbf{d}$ The average swimming areas of control, Slit2-control-MO and Slit2-MO are $126.59 \mathrm{~cm}^{2}, 108.92 \mathrm{~cm}^{2}$ and $16.40 \mathrm{~cm}^{2}$ respectively. e The average swimming distances of control, Slit2-miss-control and Slit2-MO are $67.44 \mathrm{~cm}, 64.24 \mathrm{~cm}$ and $23.83 \mathrm{~cm}$ respectively. $\mathbf{f}$ The average swimming speeds of control, Slit2-control-MO, and Slit2-MO are $2.28 \mathrm{~cm} / \mathrm{s}, 2.06 \mathrm{~cm} / \mathrm{s}$ and $0.82 \mathrm{~cm} / \mathrm{s}$, respectively. j The average swimming areas of control, Robo2-control-MO, and Robo2-MO are $126.59 \mathrm{~cm}^{2}, 110.84 \mathrm{~cm}^{2}$ and $19.42 \mathrm{~cm}^{2}$, respectively. $\mathbf{k}$ The average swimming distances of control, Robo2-control-MO, and Robo2-MO are $67.44 \mathrm{~cm}, 59.24 \mathrm{~cm}$ and $21.99 \mathrm{~cm}$, respectively. I The average swimming speeds of control, Robo2-control-MO, and Robo2-MO are $2.28 \mathrm{~cm} / \mathrm{s}, 1.94 \mathrm{~cm} / \mathrm{s}$ and $0.83 \mathrm{~cm} / \mathrm{s}$, respectively. Error bars are shown as standard deviation (SD). Data denoted by the same letter are not significantly different $(P>0.05)$ by Scheffé test after one-way analysis of variance

genes in developing Xenopus embryos. At the early tailbud stage (stage 32), Xlslit2 was expressed in the alar plate throughout the telencephalon, diencephalon, and mesencephalon (Fig. 4a). However, Xlslit2 expression domain in the dorsal diencephalon represented discontinuous pattern with many Xlslit2-weak gaps. One of these gaps (arrow in Fig. 4a') appeared to be corresponded to the position of the posterior commissure (see below). $X l s l i t 2$ was also located in the ventral region of the mesencephalon. In the metencephalon, Xlslit2 expression domains manifest as a series of clusters located along the anteroposterior axis (arrowheads in Fig. 4a), one of which may correspond to the facial motor nucleus, as shown for Slit2 expression in mouse [42]. At the middle 

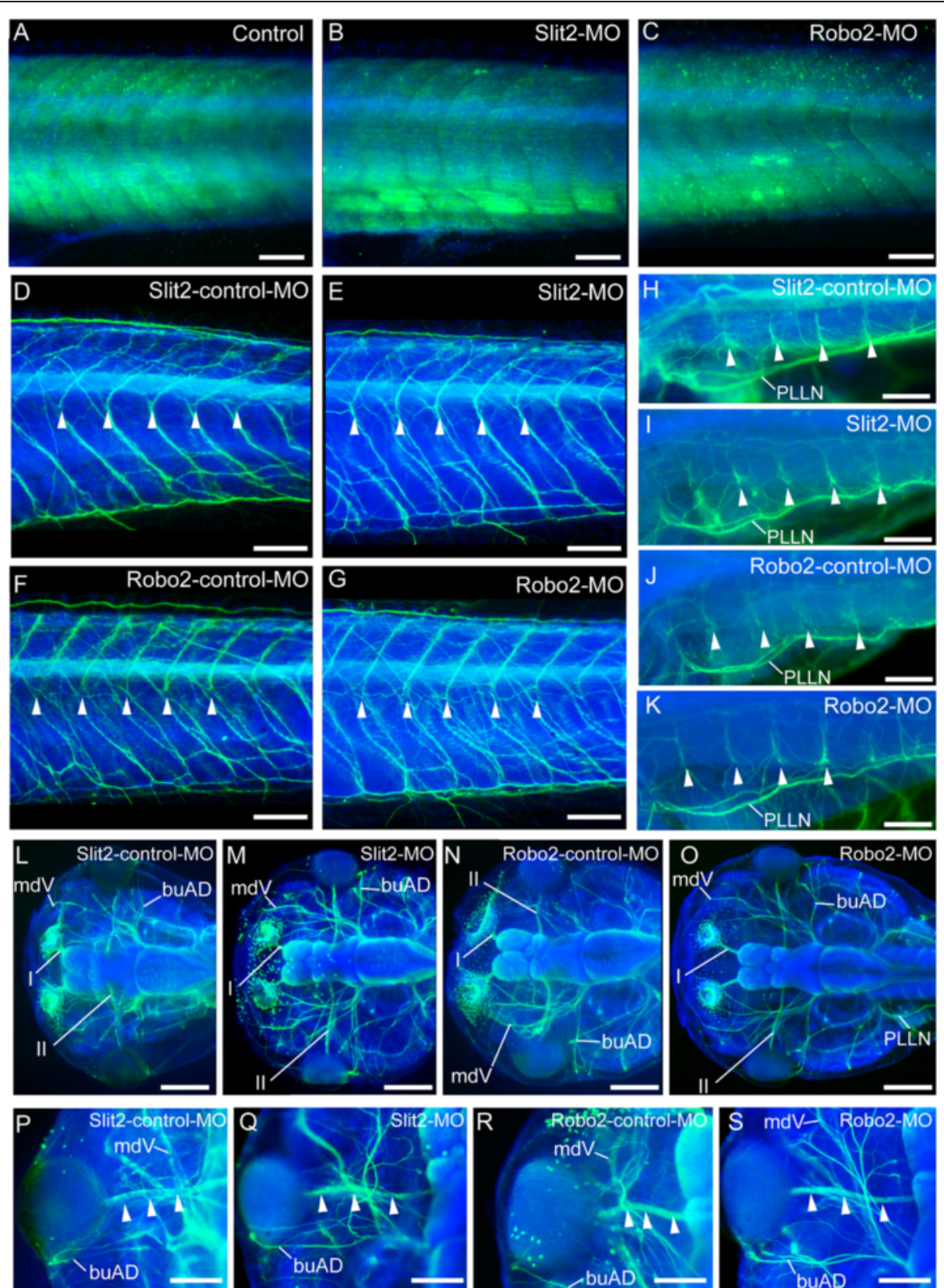

Fig. 3 External morphology of Xenopus larvae. Blue staining show nucleus labeled by DAPI. Myotomes (a-c) and nerves (d-s) are visualized by immunohistochemistry (shown in green). a-c Lateral view of the trunk in un-injected control (a), slit2-MO-injected (b) and robo2-MO-injected (c) specimens. Development of myotomes is normal in all conditions. $\mathbf{d}-\mathbf{g}$ Lateral view of the trunk in slit2-control-MO-injected (d), slit2-MOinjected (e), robo2-control-MO-injected (f), robo2-MO-injected (g) specimens. Arrowheads indicate segmentally organized spinal nerves. h-k Dorsal view of the anterior trunk in slit2-control-MO-injected (h), slit2-MO-injected (i), robo2-control-MO-injected (j), robo2-MO-injected (k) specimens. Arrowheads indicate segmentally organized spinal nerves. I-o Dorsal view of the head in slit2-control-MO-injected (I), slit2-MO-injected (m), robo2-control-MO-injected (n), robo2-MO-injected (o) specimens. p-s Dorsal view of the optic and cranial nerves in slit2-control-MO-injected (p), slit2-MO-injected (q), robo2-controlMO-injected (r), robo2-MO-injected (s) specimens. Arrowheads indicate the optic nerves. The peripheral nerves visualized by immunohistochemistry represent a normal innervation pattern. Scale bars: A-K, P-S, $200 \mu \mathrm{m} ; \mathrm{L}-\mathrm{O}, 500 \mu \mathrm{m}$

tail-bud stage (stage 40), Xlslit2 was expressed in the alar plate of the telencephalon and diencephalon, as in stage 32 (Fig. 4b). However, the expression level observed in the dorsal alar plate decreased at the dorsal midbrain; thus, the posterior limit of the expression domain was restricted at the posterior diencephalon (arrow in Fig. 4b).

In Xenopus embryos at late tail-bud stage (stage 44), Xlslit2 transcripts were expressed at high levels in the dorsal part of the diencephalon, mesencephalon, and 

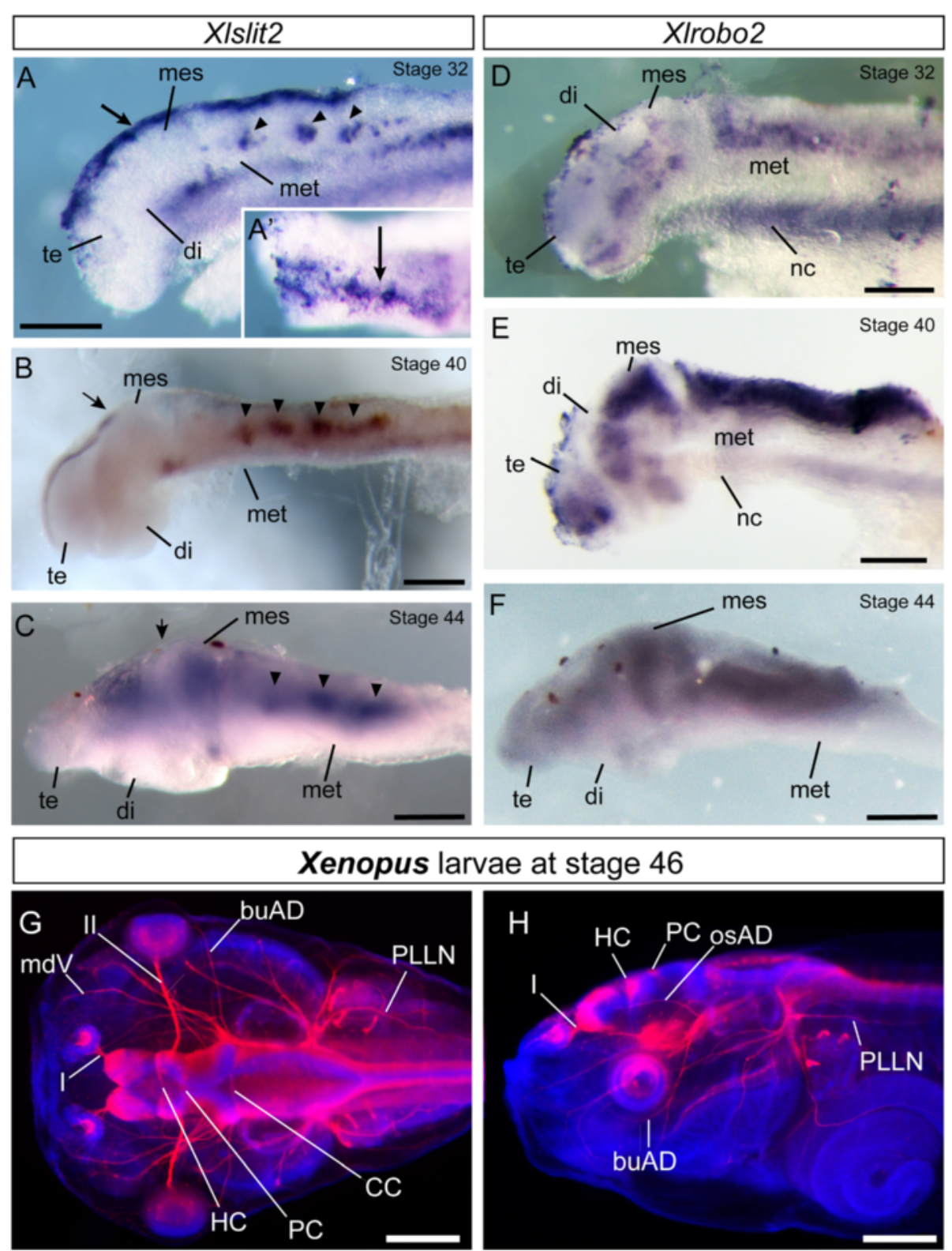

Fig. 4 Expression patterns of XIslit2 and XIrobo2 and morphology of axonal tracts in Xenopus larvae. a-c Transcripts of XIslit2 are detected through the dorsal midline in the diencephalon at stage 32 (a), stage 40 (b) and stage 44 (c). Xlslitz-weak gap is found between diencephalon and mesencephalon (arrows in A and C, $A^{\prime}$ is a dorsal view). In the metencephalon (met), expression domains of XIslit2 are observed along the antero-posterior axis (arrowheads). d-f Expression pattern of Xlrobo2 at stage 32 (d), stage 40 (e) and stage 44 (f). (d) At early tail-bud stage, Xlrobo2 is expressed in the telencephalon (te), diencephalon (di), mesencephalon (mes), dorsal metencephalon (met) and notochord (nc). e, f At middle and late tail-bud stage, XIRobo2 expression is detected at high levels in the dorsal CNS. $\mathbf{g}$, $\mathbf{h}$ Dorsal $(\mathbf{g})$ and lateral $(\mathbf{h})$ view of the developing Xenopus larva at stage 46. Axons in the PNS and CNS are visualized by anti-acetylated tubulin antibody. Habenular and posterior commissures are located the diencephalon (HC and PC). Scale bars: A-F, 200 um; G and $\mathrm{H}, 500 \mu \mathrm{m}$

metencephalon (Fig. 4c). At this stage, Xlslit2 expression regions became broader compared with those of stage 40 embryos, although the Xlslit2 expression domain was still restricted to the specific part of the metencephalon (Fig. 4c). Conversely, Xlslit2 transcripts were weakly expressed in the transitional region between the diencephalon and mesencephalon (arrow in Fig. 4c).

\section{Expression of XIrobo2 in Xenopus larvae}

Next, we observed the expression pattern of the Xenopus ortholog of robo2 (Xlrobo2), which is a putative receptor of Xlslit2. At stage 32, Xlrobo2 was expressed at high levels in the metencephalon (Fig. 4d). Furthermore, the Xlrobo2 transcript was observed in the diencephalon and ventral telencephalon (Fig. 4d). At stage 40, the 
Xlrobo2 mRNA was detected throughout the dorsal level of the brain (Fig. 4e). In particular, it was expressed in the dorsal sides of the mesencephalon and metencephalon, whereas it was weakly expressed in the ventral neural tube. We did not detect Xlrobo2 transcripts in the spinal cord (data not shown). At stage 44, Xlrobo2 was expressed at higher levels throughout the dorsal part of the brain, as in the previous stage (Fig. 4f).

\section{Morphology of the nervous system in Xenopus larvae}

Immunostaining using an anti-acetylated tubulin antibody was performed to investigate the developmental process of the Xenopus nervous system. In the embryonic stage 46 , several cranial nerves, including the olfactory (the first cranial nerve, I), the optic (the second cranial nerve, II), the trigeminal $(\mathrm{mdV})$, the anterior lateral line (buAD), and the posterior lateral line (PLLN) nerves, were observed (Fig. 4g, h), as in matured tadpole larvae. We also identified spinal nerves arranged segmentally in the trunk region (data not shown). These nerves showed clear segregation and projected to their correct targets. Next, we studied the axonal organization of the CNS, which receives input from the peripheral nerves. At stage 46, we observed several longitudinal or commissural axonal bundles, in which three distinct commissural tracts were observed in the dorsal side of the neural tube (Fig. 4g, h). Among those, the HC was located in the anterior part of the dorsal diencephalon. The PC was observed in the posterior part of the dorsal diencephalon (apparently corresponding to the pretectum). The commissures in the cerebellum (CC), which may include commissure cerebelli and commissure vestibulolateralis [43], were located on the cerebellum and across the midline on the dorsal side.

\section{Expression of XIslit2 and XIrobo2 in relation to the commissural tracts}

We then compared the expression domains of Xlslit2 and Xlrobo2 in immunostained axon tracts, to determine whether Xlslit2 and Xlrobo2 expression domains correspond to the tracts of early-developing axons reported previously in Xenopus [1, 44, 45]. We initially studied the expression pattern of Xlslit2 at stage 42. In this stage, Xlslit2 transcripts represent a discontinuous expression pattern in the transitional region between the diencephalon and mesencephalon, where its expression domain is located in a slightly deeper region while its anterior limit reached to the superficial part (Fig. 5a, d, arrow in Fig. 5d'). Double labeling using DIG-labeled Xlslit2 probe and anti-acetylated tubulin antibody revealed that the position of the Xlslit2 expression domain was localized beneath the axon bundle of the PC, which crosses the midline on the dorsal diencephalon (Fig. 5b, c, e, f). It appears that PC axons path through Xlslit2 weak region in the expression domain and its anterior end corresponds to the anterior limit of Xlslit2 expression domain (Fig. 5a, c, c', arrow in Fig. 5f'). In addition, the Xlrobo 2 mRNA was located in the ventral side of the $\mathrm{PC}$, where axons contributing to the $\mathrm{PC}$ appear to originate. Thus, to clarify the anatomical position of Xlrobo2-expressing nuclei, we performed neuron labeling in the larval brain. As noted above, $\mathrm{HC}$ and PC were found on the anterior and posterior side of the dorsal diencephalon, respectively (Fig. 4g). Thus, NeuroVue was injected into the dorsal diencephalon, where $\mathrm{HC}$ and PC are thought to be located. We observed the retrogradely labeled axons of $\mathrm{HC}$ and $\mathrm{PC}$ in stage 45 larvae (Fig. 6a, b), and identified the labeled cell bodies in the section of the pretectum (posterior diencephalon). Comparison with adult brain morphology, the crop of cell bodies was thought to correspond to the nucleus of the tract of the posterior commissure (nTPC), which sends axons into the PC in many vertebrates [46] (Fig. 6b, b').

Next, to identify the positional relationship between the expression domain of Xlrobo 2 and the nTPC, stained specimen were cut into coronal sections and were observed at stage 40 . In the anterior diencephalon (presumptive thalamus), the expression domain of Xlrobo2 was located in the ventral side, and it was not detected in the dorsal area, including the habenular nucleus (Fig. 6c). In contrast, in the section of the posterior diencephalon (pretectum), the Xlrobo2 transcript was located in the dorsal part, corresponding to the nTPC (Fig. 6d, asterisk).

\section{Morphology of the developing CNS in MO-injected larvae}

To identify morphological phenotypes of Xlslit2- and Xlrobo2-MO-injected larvae in the developing nervous system, we observed the overall morphology of the larval CNS in MO-treated specimens, and found a normal morphology for the telencephalon, diencephalon, mesencephalon, and metencephalon in both control-MO- and Xlslit2-MO- or Xlrobo2-MO-treated larvae at stage 44 (Fig. 7a, b, d, e). Next, to clarify whether some defects could be detected in the neuronal circuit, we observed the morphology of commissural tracts $(\mathrm{HC}, \mathrm{PC}$, and $\mathrm{CC})$ on immunostained specimens. We found that the morphologies of the $\mathrm{HC}$ and $\mathrm{CC}$ in larvae treated with slit2 or robo $2 \mathrm{MO}$ were not significantly different from those of the control-MO-treated larvae, whereas the PC in Xlslit2-MO or Xlrobo2-MO-treated larvae exhibited an unclear commissural bundle on the dorsal diencephalon (Fig. 7b, e). This may be explained by the defasciculation of the tract, so that it could not be observed as a tight bundle. Thus, we then measured the width of bundles, and found that the PC in Xlslit2-MO-or Xlrobo2MO-treated larvae was significantly wider than that of 

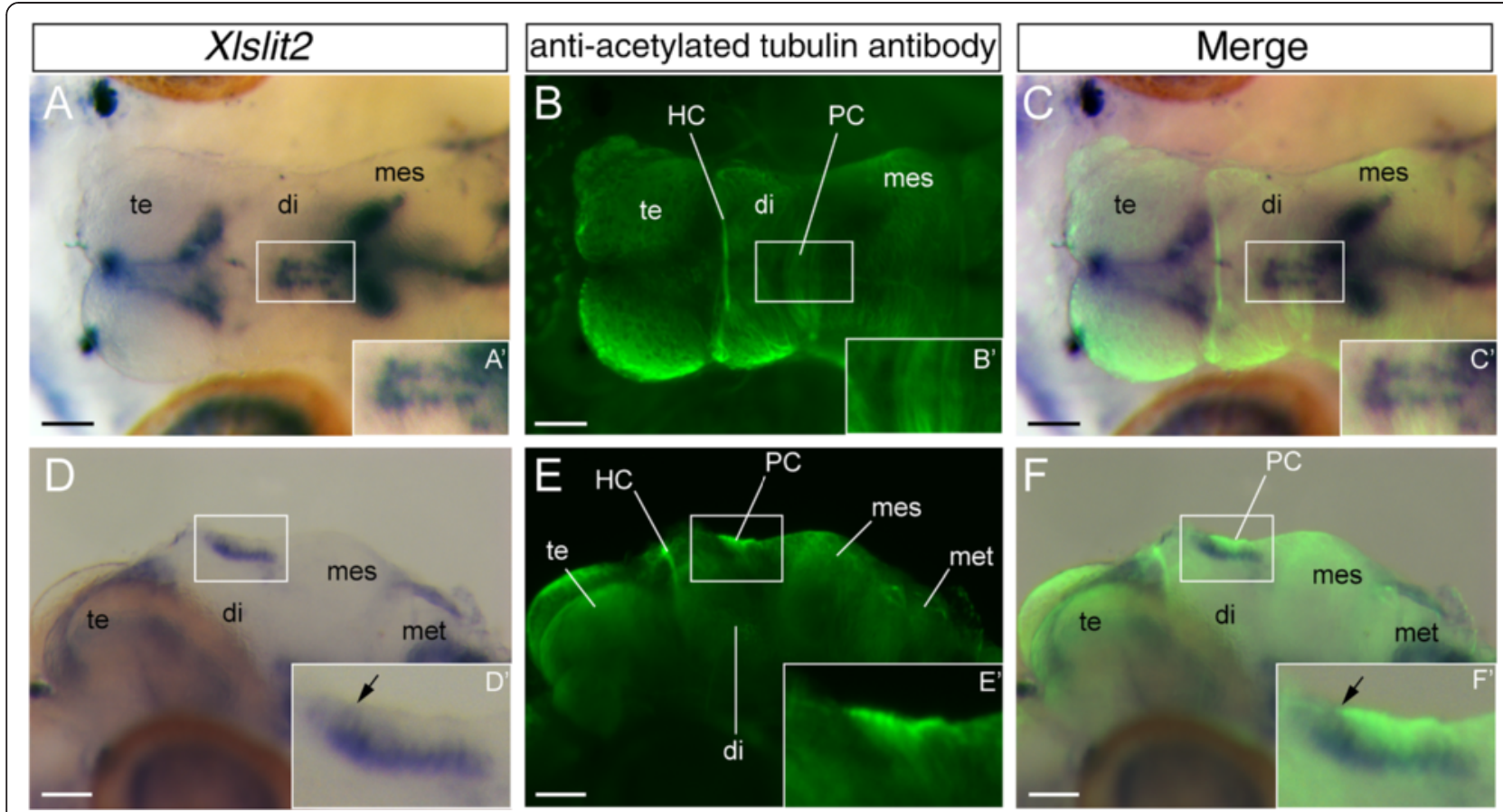

Fig. 5 Morphology of axonal tracts and X/slit2 expression domain in the neural tube. Dorsal (a-c) and lateral (d-f) view of the developing Xenopus larva at stage 42. ( $\left.\mathrm{A}^{\prime}-\mathrm{F}^{\prime}\right)$ High-magnification images of white squares in A-F. a, c Transcripts of XIslit2. XIslit2 mRNA is discontinuously expressed in the dorsal diencephalon. The anterior limit of XIslit2 attach to the superficial region of the brain (arrow in D). $\mathbf{b}$, e Axons in the CNS are visualized by immunohistochemistry (shown in green). Habenular and posterior commissures are visible. c, f Merged image of Xlslit2 (purple) and nerves (green). The anterior end of the PC corresponds to the anterior limit of X/slit2 expression domain (arrow in F). Scale bars: $100 \mu m$

control larvae, whereas the width of the $\mathrm{HC}$ and $\mathrm{CC}$ was unaffected (Fig. 7c, f).

\section{Morphology of the PC in MO-treated embryos}

To clarify further the defect of the PC, we studied the morphology of the $\mathrm{PC}$ in coronal sections of embryos at stage 44 and found that the PC bundle in Xlslit2-MOor Xlrobo2-MO-treated larvae became thinner on the dorsal diencephalon compared with those of control specimen (Fig. 8). Similarly, in the sagittal sections, the PC in Xlslit2-MO- or Xlrobo2-MO-treated larvae became thinner in dorso-ventral axis, and exhibited an anteroposteriorly elongated morphology (Fig. 9).

Overall, the results of this series of experiments suggest that Slit2 and/or Robo2 is specifically involved in the fasciculation of the PC.

\section{Discussion}

In the present study, we focused on the function of Slit2 and Robo2, both of which play crucial roles in the patterning of CNS axons [47], and identified three novel findings. First, expression patterns of Slit2 and Robo2 are spatiotemporally regulated. Second, slit2-MO- and robo2-MO injected larvae exhibited abnormal swimming behavior. Third, slit2-MO- and robo2-MO-injected larvae showed an abnormal morphology of the $\mathrm{PC}$, a part of the embryonic axonal tract. These results suggest that the Slit2-Robo2 interaction primarily or secondarily affects to the neuronal circuitry that triggers the coordinated swimming pattern.

\section{Involvement of Slit2 and Robo2 in swimming behavior}

We found that larvae treated with slit2 and robo2 $\mathrm{MO}$ exhibited an abnormal swimming trajectory. In addition, the swimming distance and speed of MO-injected larvae were also reduced, although control-MO-injected larvae showed no apparent defects in swimming behavior. We also found that slit2-MO- and robo2-MO-treated larvae showed a normal body musculature. These data suggest that Slit2 and Robo2 inhibition induced the abnormal swimming pattern observed, without disturbing basic body apparatuses, such as the fins or body muscles. Importantly, as noted above, both Xlrobo2-MO- and control-MO-treated larvae included specimens that exhibited abnormal morphology of the head and eye. Despite the presence of these head abnormalities in both control-MO- and robo2-MO-treated larvae, we observed a significant abnormality in Xlrobo2-MO-treated specimens regarding swimming behavior, whereas no significant problem was detected in the control group. Given that MOs were specifically injected into blastomeres, which differentiate into the nervous system, we speculated that 

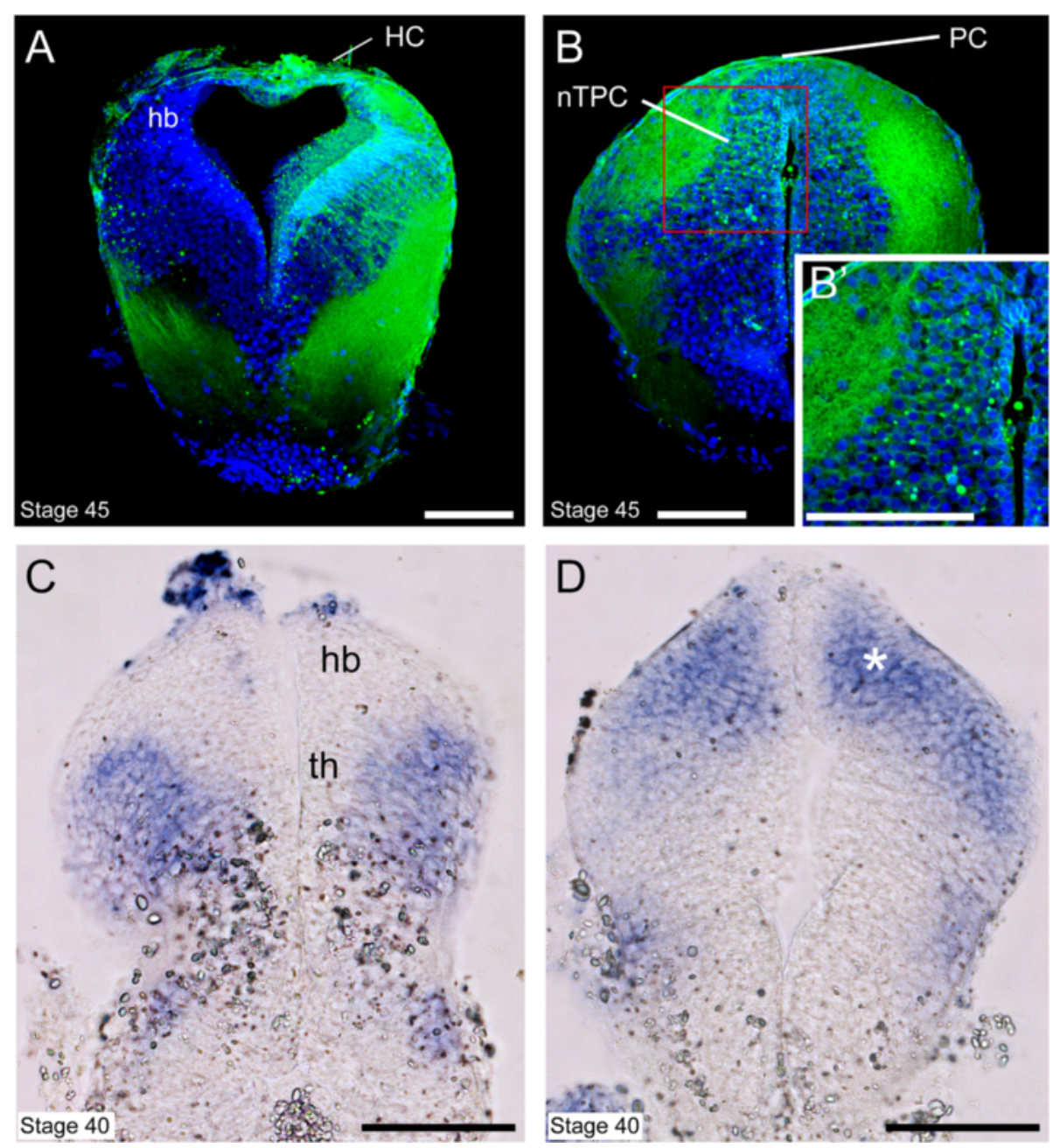

Fig. 6 Morphology of axonal tracts and XIrobo2 expression domain in the diencephalon. $\mathbf{a}$, b Coronal sections of the tadpole brain at stage 45, in which NeuroVue chips were inserted on the dorsal diencephalon and labeled neurons are shown in green. Blue staining shows nucleus labeled by DAPI. a Labeled axons (green), which correspond to the HC, are observed in the dorsal region of the diencephalon. $\mathbf{b}$ Labeled PC axons are observed in the dorsal diencephalon and labeled cell bodies are located ventral to the PC. This region is the presumptive nucleus of the tract of the PC (nTPC). ( $\mathrm{B}^{\prime}$ ) High-magnification image of the red box in B. $\mathbf{c}$, d Expression pattern of XIrobo2 on coronal sections of the tadpole brain at stage 40. $\mathbf{c}$ and $\mathbf{d}$ are the slice of anterior and posterior diencephalon, respectively. $\mathbf{d}$ XIrobo2 expression domain is observed in the nTPC (asterisk). Scale bars: $100 \mu \mathrm{m}$

MO-injected larvae exhibited nervous system defects in circuits that control body movement.

\section{Peripheral nerves in MO-treated larvae}

We found that larvae treated with slit2 and robo2 $\mathrm{MO}$ exhibited an apparent normal morphology in the peripheral nerves including cranial and spinal nerves. Although one previous report indicated that central projection of the optic nerve in zebrafish robo2 mutant was abnormal [48], we did not find any abnormality of the optic nerve bundle projecting to the brain. However, as we could not follow the trajectory of the optic nerve toward the optic tectum, further experiment by axon labeling will be needed to identify whether MO-treated larvae exhibit abnormalities in the optic tract.

\section{Expression of slit2 and robo2 in developing Xenopus larvae}

To clarify whether MO-treated specimens have any problem in the neurodevelopmental process, we studied the expression pattern of the slit 2 and robo 2 transcripts in relation to the developmental position of the commissural tracts.

Previous studies have showed that the Slit2 and Robo2 mRNAs are expressed in the developing nervous system in many vertebrates [21-24, 27-30]. The present study showed that Xlslit2 was expressed in a specific part of the developing CNS, as in other vertebrates [23, 24, 49, 50]. We found that the expression domains of Xlslit2 and Xlrobo2 corresponded to the region in which the PC is formed; the Xlslit2 expression domain located beneath 


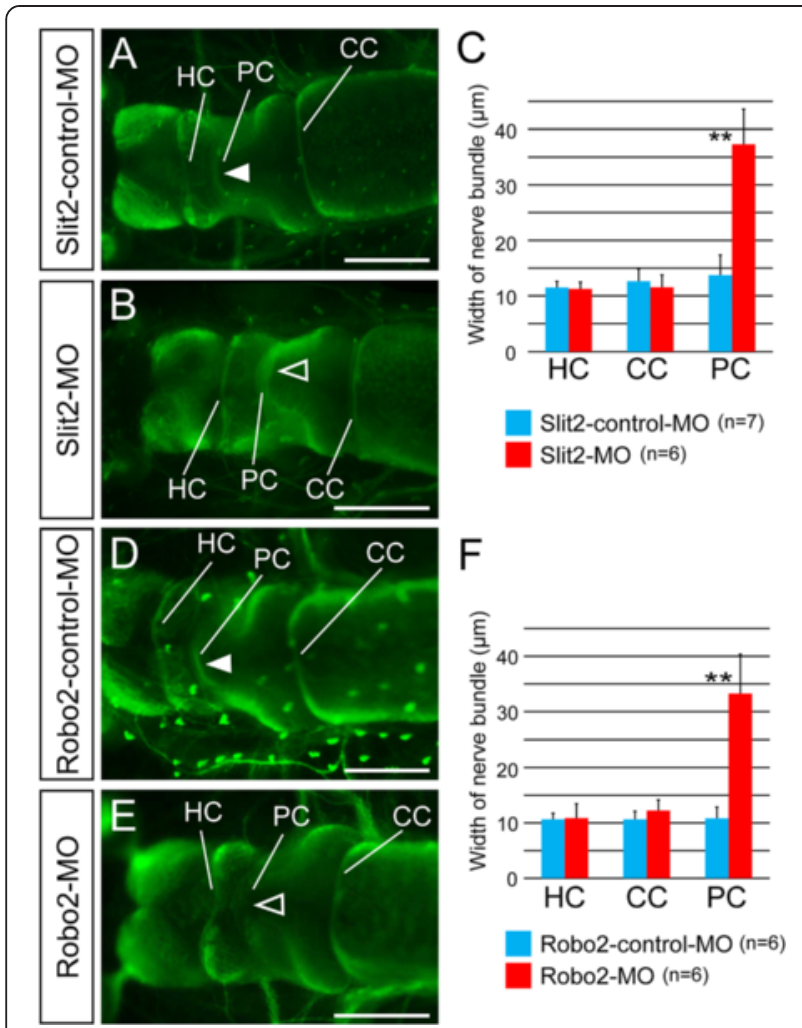

Fig. 7 slit2/robo2-MO injected larvae change the width of the posterior commissure. $\mathbf{a}, \mathbf{b}, \mathbf{d}$, e dorsal views of the tadpole brain at stage 44. a In slit2-control-MO injected larva, three commissures (habenular commissure: HC, posterior commissure: PC and cerebellar commissures: CC) are observed. PC indicated by an arrowhead. $\mathbf{b}$ In Slit2-MO injected larva, morphology of the habenular and cerebellar commissure appear to be normal, whereas nerve bundle of the posterior commissure become wider than the control larva (open arrowhead). c Quantification of the width of nerve bundle in Slit2control- (blue)/Slit2-MO (red). slit2-MO-injected larva is significantly changed the width of the posterior commissure compared to that of slit2-control-MO (**P $<0.01$ ). $\mathbf{d}$, e In robo2-control- (D)/robo2-MO (e) injected larvae, the former represents three commissures but the latter shows unclear PC bundle (open arrowhead). $\mathbf{f}$ Quantification of the width of three commissures. The posterior commissure of robo2MO-injected larva is significantly changed the width of the PC. Error bars are shown as standard deviation (SD). *P value was obtained by ANOVA ( $P<0.05$ is significant). ( $\left.{ }^{*} P<0.01\right)$. Scale bars: $200 \mu \mathrm{m}$

the PC, and Xlrobo2 was expressed in the nTPC, which may be a source of the PC (see below). Importantly, $X l s l i t 2$ domain showed a discontinuous pattern in which PC appears to be extended in Xlslit2-weak region, and the anterior limit of Xlslit2 expression domain corresponds closely to the anterior border of the PC. In addition to the spatial distribution, the temporal timing of Xlslit2/Xlrobo2 expression was though to correspond to the development of the PC. Namely, the early scaffold of Xenopus laevis is formed at stage 32 [1], a time at which the PC (called TPC in Xenopus) is across the midline of the dorsal diencephalon. Our study showed that the expression of Xlslit2 and

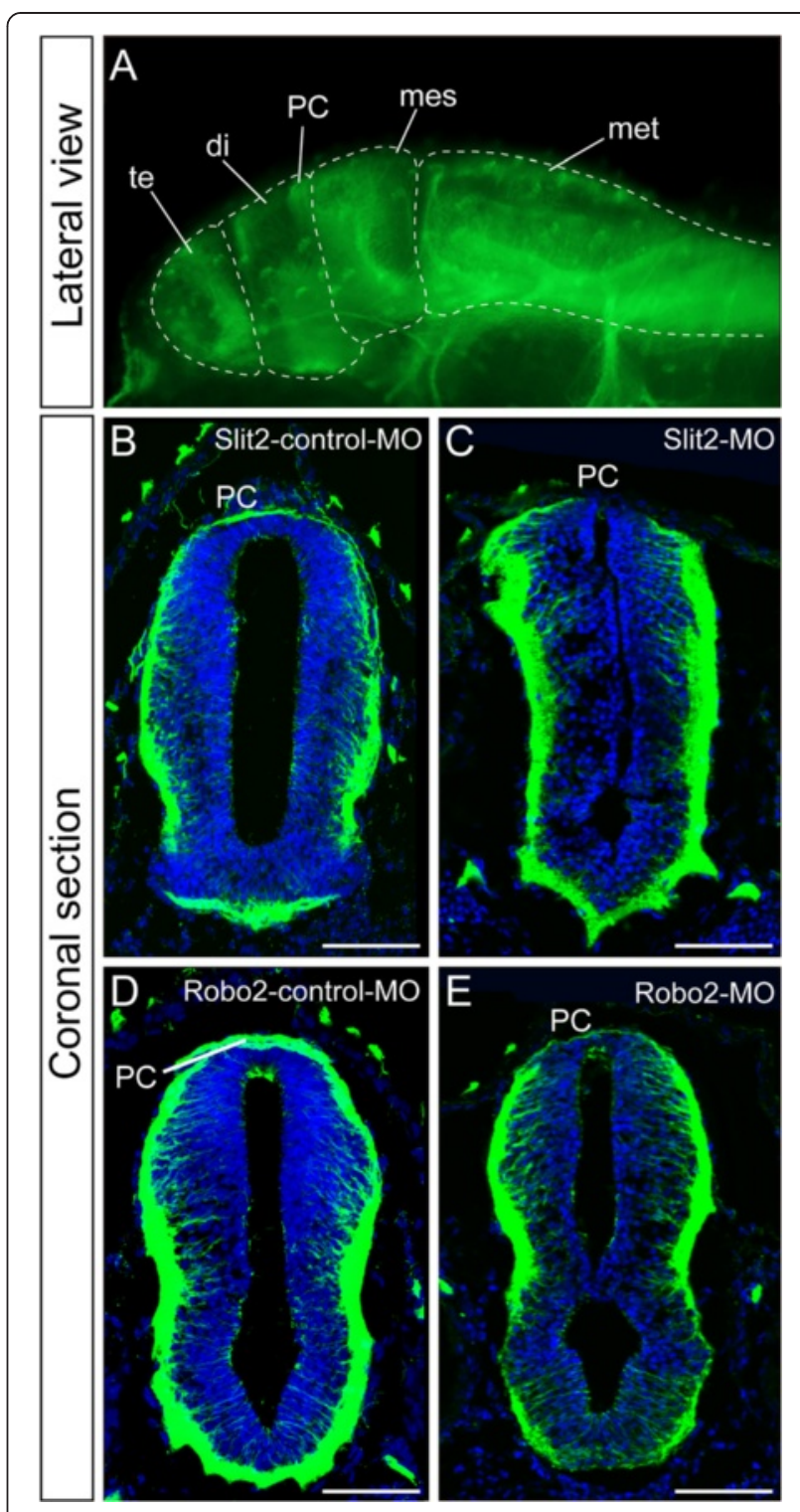

Fig. 8 slit2/robo2-MO injected larvae represent the abnormal morphology of the posterior commissure. Neurons are visualized by immunohistochemistry (shown in green). a Lateral view of the brain in Xenopus larva. Dashed line indicates the outline of the brain regions. b-e Coronal sections at the level of the posterior commissure: $\mathbf{b}$ Slit2control-MO, c Slit2-MO, d Robo2-control-MO and e Robo2-MO. In slit2/ robo2-MO-injected larva, the bundle of the posterior commissure is thinner than that of control-MO. Blue staining show nucleus labeled by DAPI. Scale bars: 100 um

Xlrobo2 was observed at stage 32, which corresponds to the onset of PC formation. Xlslit2 and Xlrobo2 were also expressed at stage 40, a time point at which the early tract is being constructed. Subsequently, the Xlslit2 and Xlrobo2 transcripts were observed at stage 44, a stage at which tight bundle of PC could be observed. Thus, Xlslit2 and Xlrobo2 were expressed correspondingly to the developmental time course of the PC. 

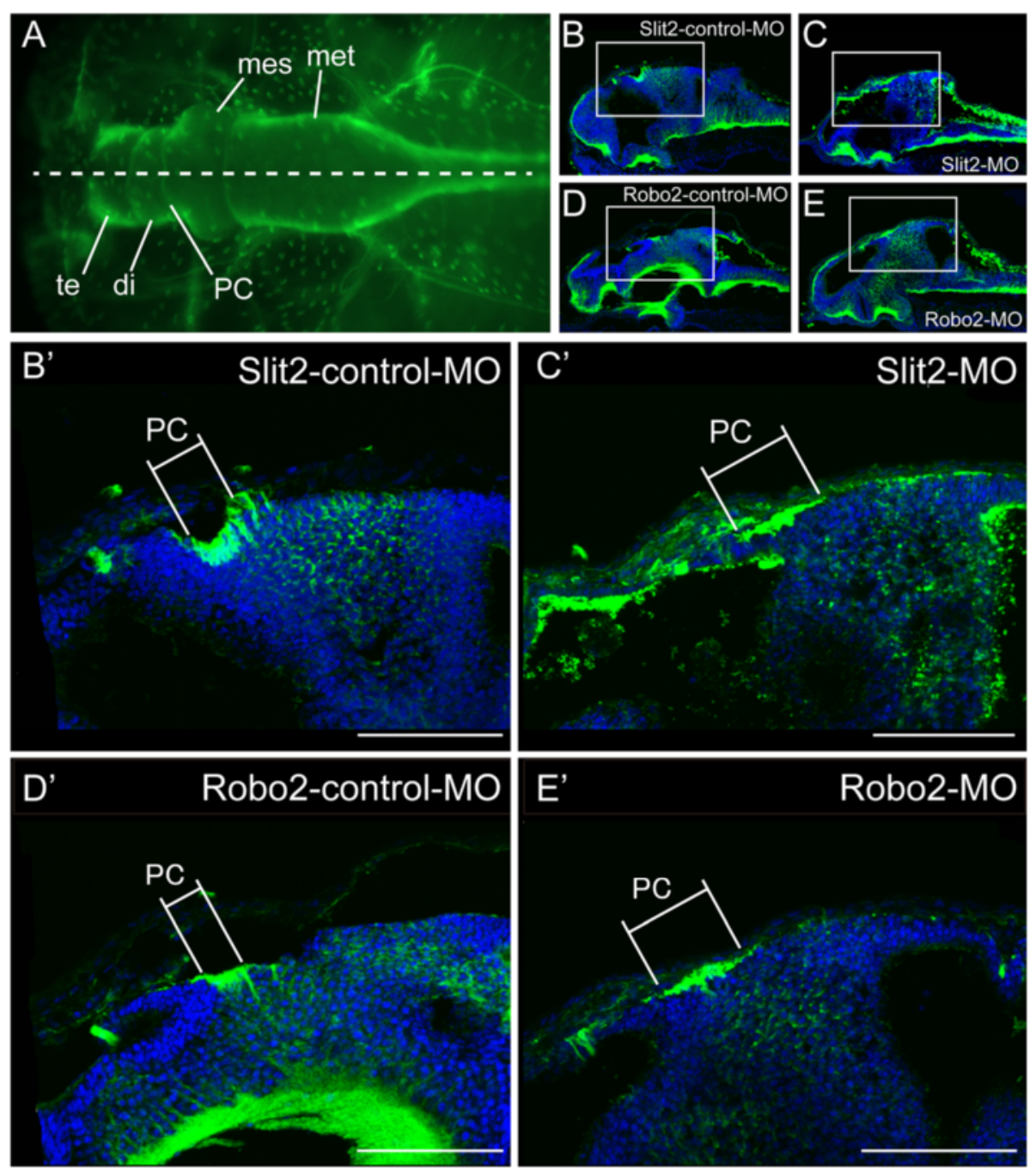

Fig. 9 Median sections of the tadpole brain. Neurons are visualized by immunohistochemistry (shown in green). a Dorsal view of the Xenopus larva that performed the fluorescent immunostaining. Dashed line indicates the cutting plane in (b-e). $\mathbf{b}^{\prime}-\mathbf{e}^{\prime}$ High magnification images of the box in (b-e): b, b' Slit2-control-MO, c, c' Slit2-MO, d, d' Robo2-control-MO, e, e' Robo2-MO. In the Slit2/Robo2-control-MO, the posterior commissures make a tight bundle, whereas in the Slit2/Robo2-MO injected larvae, the width of PC is elongated anteroposteriorly. Blue staining shows nucleus labeled by DAPI. Scale bars: 100 um

\section{Interaction between slit2 and robo2 in the formation of} the PC

The present study revealed that, although the $\mathrm{HC}$ and CC showed normal morphology, the PC exhibited an abnormal morphology in both slit2-MO- and robo2MO-injected larvae. This may be due to a problem in the fasciculation process; the PC bundle in MO-treated specimens was thinner and wider compared with that of control larvae. Previous studies using insects and vertebrates showed that Slit2 and Robo2 are involved in the formation of axon bundles [22, 23] and commissural tracts [25-30, 32]. Those findings support our present finding that Xenopus cognates of Slit2 and Robo2 seem to be involved in the formation of the PC.
Regarding the molecular mechanism underlying PC formation in Xenopus, the present study showed that the Xlrobo2 expression domain in the diencephalon appeared to correspond to the region that includes the nTPC. Because slit2-MO- and robo2-MO-injected larvae showed a very similar phenotype, XISlit2 may act as a ligand for XlRobo2 during the formation process of the PC. Thus, it is speculated that XlRobo2-expressing PC axons may avoid the XISlit2 protein secreted from the pretectum, thus allowing axons to pass through the Slit2-negative or Slit2-weak region. We found that Xlslit2 mRNA exhibits discontinuous expression beneath the PC, and PC axons appear to run through an Xlslit2weak region. This morphological relationship between 
Slit2 and PC axons has not been reported in other vertebrates. This finding may thus provide new insights into the function and evolution of Slit-Robo signaling. Importantly, anterior domain of Xlslit2 extended dorsally and attached to the superficial region of the brain where the anterior border of the PC was formed. In the slit2MO-injected larvae, the anterior border of PC seemed to be extended toward the anterior neural tube, which suggests that the PC axons are repelled by XISlit2 in the superficial region of the diencephalon, resulting in a tight bundle on the posterior side of the pretectum (Fig. 10); however, the manner in which the posterior limit of the tract is formed remains unknown. Importantly, despite the abnormalities in the PC in MO-treated embryos, we found a normal morphology for the other commissures (HC and CC) in Xlslit2-MO- and Xlrobo2MO-treated embryos. This may be attributable to the absence of Xlrobo2 transcripts in the nuclei that provide axons to those tracts. Other guidance molecule(s) may be involved in the formation of those commissures.

\section{Evolutionary perspective of Slit-Robo signaling in the formation of the PC}

Recent studies have shown that in amniotes (chick and mouse), PC located to slit2 weak region on the dorsal diencephalon neighboring to the anterior midbrain $[23,49]$. In addition, Robo2 transcripts appear to be expressed in the region corresponding to the nTPC in mice $[23,51,52]$. These morphological characters are markedly similar to those in Xenopus laevis represented in our present study. On the other hand, although the expression domain of Slit2 cognate in zebrafish appeared to correspond to those of other amniotes [24, 53], expression pattern of zebrafish Robo2 seems to be different from those of amniotes [54]. Namely, its transcripts appeared to be absent in the nTPC [22]. Taken together, origin of Slit -Robo signaling that contributes to the PC formation may date back to the common ancestor of tetrapods (Fig. 11). However, abnormality in the PC has not been reported in other amniotes including slit or robo mutant mice $[27,28,30]$. It may be due to the fact that the disruption of slit or robo signaling may be compensated for by other paralogues or guidance molecules, such as the EphA7-dependent system which is involved in the formation of the chick PC [55]. Thus, further functional studies using vertebrate groups should be necessary to identify the evolutionary process of PC formation. Consequently, Slit2-Robo2 signaling might contribute to the generation of the novel commissural system which participated in the evolution of higher cognitive center, as Slit2-Robo2 signaling is involved in the formation of corpus callosum (cc; [28]), a well developed commissural system, which thought to be newly established in eutherian mammals. Since the cc plays a key role in the cognitive function in the mammalian neocortex,

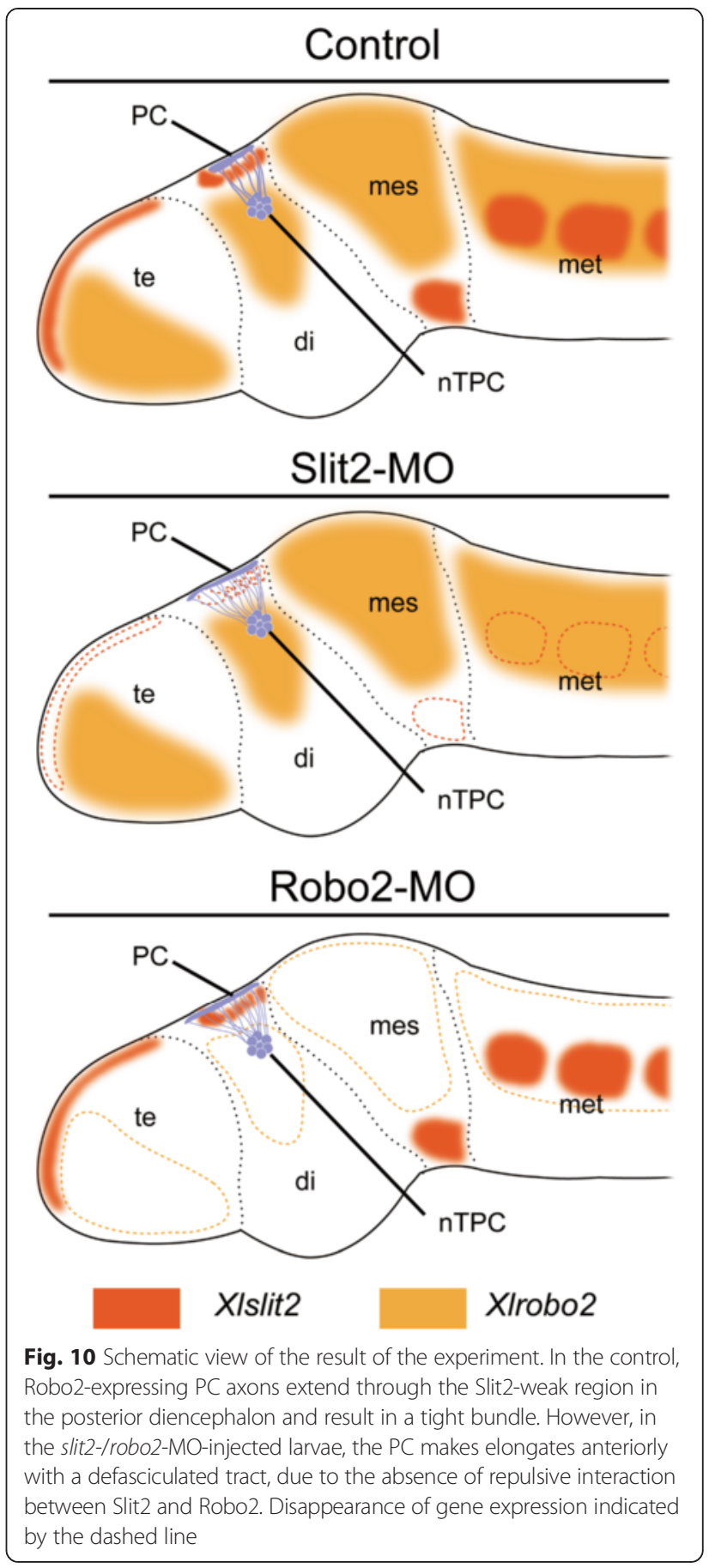

establishment of cc may be an important step in the evolution of the neocortex as the highest integrative center.

\section{Neuronal defects may contribute to abnormal behavior}

It is reasonable to speculate that, if Xlslit2-MO- and Xlrobo2-MO-treated larvae exhibit defects in the neuronal circuitry of the CNS, they would show some kind of defect in motor movement, due to the disruption of 


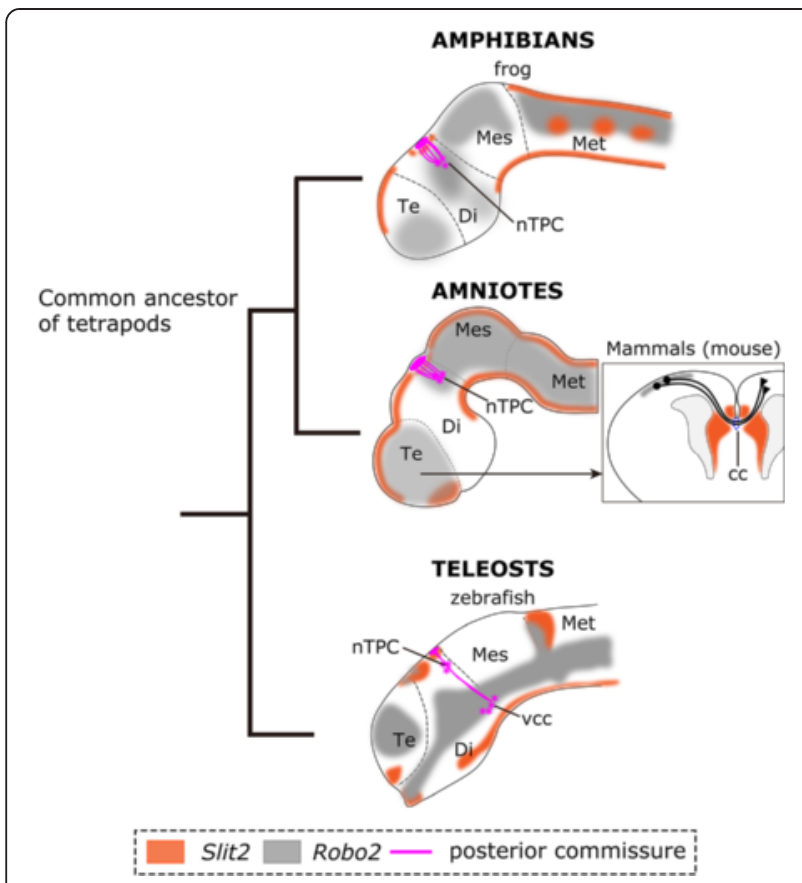

Fig. 11 Evolutionary process of Slit/Robo mediated axonal wiring. In amphibians and amniotes, Slit2-weak region is present in the superficial part of the diencephalon, in which posterior commissure is formed. Robo2 2 is expressed in axons of the posterior commissure originates from $\mathrm{NTPC}$ in the posterior diencephalon. In the zebrafish ( $24 \mathrm{~h}$ post fertilization), slit2 expression domain in the dorsal diencephalon is similar to those of tetrapods, whereas robo2 transcripts are appear to be absent in the homologous region of nTPC. In mammals, Slit/Robo is also used for the development of the newly acquired commissure, such as corpus callous (indicated by the blue dashed ring). cc, corpus callous; Di, diencephalon; Mes, mesencephalon; Met, metencephalon; nTPC, nucleus of the tract of the posterior commissure; PC, posterior commissure; Te, telencephalon; vcc, ventrocaldal cluster neuronal wiring. In fact, we found that Xlslit2-MO and Xlrobo2-MO-injected larvae showed an abnormal swimming pattern. As this behavior is thought to be an initial form of free swimming in Xenopus larvae, it may be controlled mainly by a neuronal circuit constructed by an intrinsic neurodevelopmental program. Thus, the SlitRobo interaction appears to be involved in the establishment of functional brain element(s) that are formed in the embryonic or early larval stage and regulate an initial free swimming. Because these molecules are expressed at early stages, this behavioral defect may originate from the disruption of an early developing neuronal circuit that induces the coordinated swimming pattern. Otherwise, the later-developing axons that follow earlydeveloping tracts constructed by Slit2-Robo2 signaling may contribute to the behavioral problem detected. Previous studies showed that inhibition of Slit2 or Robo2 results in an abnormal morphology of the TPOC in zebrafish and mouse [21-23]. Thus, Xlslit2-MO- and Xlrobo2-MO-injected Xenopus embryos may develop abnormalities in the TPOC itself, as in other vertebrates, which may cause behavioral abnormalities. Otherwise, the neuronal circuits that are formed subsequently on the framework of the TPOC may be related to the behavioral problem observed in the present study, although we were unable to check abnormality in the TPOC using these morphants due to technical difficulties (unavailability of specific antibodies that recognize TPOC). In addition, the commissural interneurons in the spinal cord that produce appropriate muscle contraction and body movements may be affected by Xlslit2-MO- and Xlrobo2 inhibition, although transcripts of Xlslit2 and Xlrobo2 could not be observed in trunk myomeres and the spinal cord, respectively. However, as observed in the present study, abnormal morphology of the PC may induce behavioral changes. Although it is unknown whether there is a direct link between the PC and swimming behavior, it is important to note that axons in the PC stem from several types of neurons. Previous studies have shown that the PC contains axons that stem from several nuclei, including the nTPC $[46,55]$. In mammals, the PC is involved in oculomotor movement by transmitting visual information coming from the cerebral cortex (visual area) and superior colliculus [56, 57]. Moreover, in mammals and birds, the PC contains neurons from the medial longitudinal fascicle (MLF), which includes a neuronal circuit involving the coordination of sensory and motor nerve integration. In chicks, neurons consisting of the PC (TPC) were located within the MLF, intermingled with the central and dorsal populations of MLF neurons [58]. These data suggest that the guidance mechanism for visual and/or MLF axons is affected by $\mathrm{MO}$ treatment and, hence, fails to form functional neuronal connections. Therefore, we surmise that the malformed sensory circuits in the PC would be a suitable as a candidate that caused the abnormal behavior in the slit2MO- and robo2-MO-treated larvae. Conversely, it is possible that the other neuronal system that is formed by the interaction between Slit2 and Robo2 results in the behavioral problem. Future neuroanatomical and functional studies are necessary to identify the molecular mechanism via which correct neural circuits and behavioral patterns are elicited.

\section{Abbreviations}

buAD: buccal ramus of anterodorsal lateral line nerve; CC: Commissures in the cerebellum; di: diencephalon; hb: habenular; HC: Habenular commissure; mes: mesencephalon; met: metencephalon; $\mathrm{mdV}$ : mandibular division of the trigeminal nerve; $\mathrm{mxV}$ : maxillary division of the trigeminal nerve;

nc: notochord; OSAD: superficial ophthalmic ramus of anterodorsal lateral line nerve; PC: Posterior commissure; PLLN: Posterior lateral line nerve;

te: telencephalon; th: thalamus; Sn: spinl nerve; I: olfactory nerve; II: optic nerve. Schlosser and Northcutt (2000) was referred for the morphological identification [59]. 


\section{Competing interest}

We declare that no actual or potential competing interests in relation to this article exist.

\section{Authors' contributions}

YT and KT carried out the gene expression analyses, immunohistochemical observations and the neuronal labeling, and participated in the experiments of the functional inhibition using $\mathrm{MO}$ and drafted the manuscript. MK participated in the gene isolation and expression analyses. TI carried on the immunohistochemical and behavioral analyses, and participated in the statistical assay. M. Fukagawa and YN carried on the gene expression and the immunohistochemical analyses. KTS participated in the experiments of the functional inhibition using MO. M. Fukui carried on the immunohistochemical analysis. YM conceived of the study, and participated in its design and coordination and helped to draft the manuscript. All authors read and approved the final manuscript.

\section{Acknowledgements}

We thank Dr. Mikio Inoue, Tetsuya Kominami, Kei Nakayama and Hiromi Takata for technical support and valuable discussions. We thank all past and present members of the YM laboratory for support and constructive discussions. Work in YM's laboratory was supported by RIKEN, Kobe, Japan and the Japan Society for the Promotion of Science (JSPS; grant number 24650178 and 26430018 to YM, and grant number 21770238 and 23657149 to MK).

\section{Author details}

${ }^{1}$ Graduate School of Science and Engineering, Ehime University, 2-5 Bunkyo-cho, Matsuyama 790-8577, Japan. ${ }^{2}$ Graduate School of Medicine and Pharmaceutical Sciences, University of Toyama, 2630 Sugitani, Toyama 930-0194, Japan. ${ }^{3}$ Graduate School of Science, Hiroshima University, 1-3-1 Kagamiyama, Higashi-Hiroshima, Hiroshima 739-8526, Japan.

\section{Received: 23 January 2015 Accepted: 31 August 2015}

\section{Published online: 05 October 2015}

\section{References}

1. Anderson RB, Key B. Novel guidance cues during neuronal pathfinding in the early scaffold of axon tracts in the rostral brain. Development. 1999;126:1859-68.

2. Barreiro-Iglesias A, Villar-Cheda B, Abalo XM, Anadon R, Rodicio MC. The early scaffold of axon tracts in the brain of a primitive vertebrate, the sea lamprey. Brain Res Bull. 2008;75:42-52.

3. Chitnis $A B$, Kuwada JY. Axonogenesis in the brain of zebrafish embryos. J Neurosci. 1990;10:1892-905.

4. Doldan MJ, Prego B, Holmqvist B, Helvik JV, de Miguel E. Emergence of axonal tracts in the developing brain of the turbot (Psetta maxima). Brain Behav Evol. 2000;56:300-9.

5. Easter Jr SS, Ross LS, Frankfurter A. Initial tract formation in the mouse brain. J Neurosci. 1993;13:285-99.

6. Figdor MC, Stern CD. Segmental organization of embryonic diencephalon. Nature. 1993;363:630-4.

7. Ishikawa Y, Kage T, Yamamoto N, Yoshimoto M, Yasuda T, Matsumoto A et al. Axonogenesis in the medaka embryonic brain. J Comp Neurol. 2004:476:240-53.

8. Ross LS, Parrett T, Easter Jr SS. Axonogenesis and morphogenesis in the embryonic zebrafish brain. J Neurosci. 1992;12:467-82.

9. Pike SH, Melancon EF, Eisen JS. Pathfinding by zebrafish motoneurons in theabsence of normal pioneer axons. Developmen. 1992;114:825-31.

10. Nieuwenhuys R, TenDonkelaar HJ, Nicholson C, editors. The central nervous system of vertebrates. Heidelberg: Springer-Verlag; 1998.

11. Bergquist $H$, Källén B. On the development of neuromeres to migration areas in the vertebrate cerebral tube. Act Anat. 1953;18:65-73.

12. Lumsden A, Keynes R. Segmental patterns of neuronal development in the chick hindbrain. Nature. 1989:337:424-8.

13. Orr H. Contribution to the embryology of the lizard. J Morphol. 1887;1:311-72.

14. Puelles $L$, Rubenstein $J$. Forebrain gene expression domains and the evolving prosomeric model. Trends Neurosci. 2003;26:469-76.

15. Shimamura K, Hartigan DJ, Martinez S, Puelles L, Rubenstein JL. Longitudinal organization of the anterior neural plate and neural tube. Development. 1995;21:3923-33.
16. Vaage $S$. The segmentation of the primitive neural tube in chick embryos (Gallus domesticus). Ergeb Anat Entw Gesch. 1969;4:1-88.

17. von Baer K. Über die Entwickelungsgeschichte der Thiere. Königsberg; 1828.

18. Cariboni A, Andrews WD, Memi F, Ypsilanti AR, Zelina P, Chedotal A, et al. Slit2 and Robo3 modulate the migration of $\mathrm{GnRH}$-secreting neurons. Development. 2012;139:3326-31.

19. Kennedy TE, Serafini T, de la Torre JR, Tessier-Lavigne M. Netrins are diffusible chemotropic factors for commissural axons in the embryonic spinal cord. Cell. 1994;78:425-35.

20. Rubenstein $\mathrm{J}$, Shimamura K, Martinez S, Puelles L. Regionalization of the prosencephalic neural plate. Annu Rev Neurosci. 1998;21:445-77.

21. Barresi MJ, Hutson LD, Chien CB, Karlstrom RO. Hedgehog regulated Slit expression determines commissure and glial cell position in the zebrafish forebrain. Development. 2005;132:3643-56.

22. Devine CA, Key B. Robo-Slit interactions regulate longitudinal axon pathfinding in the embryonic vertebrate brain. Dev Biol. 2008;313:371-83.

23. Ricano-Cornejo I, Altick AL, Garcia-Pena CM, Nural HF, Echevarria D, Miquelajauregui $A$, et al. Slit-Robo signals regulate pioneer axon pathfinding of the tract of the postoptic commissure in the mammalian forebrain. J Neurosci Res. 2011;89:1531-41.

24. Zhang C, Gao J, Zhang H, Sun L, Peng G. Robo2-slit and Dcc-netrin1 coordinate neuron axonal pathfinding within the embryonic axon tracts. J Neurosci. 2012:32:12589-602.

25. Kidd T, Bland KS, Goodman CS. Slit is the midline repellent for the robo receptor in Drosophila. Cell. 1999;96:785-94

26. Rothberg JM, Jacobs JR, Goodman CS, Artavanis-Tsakonas S. Slit: an extracellular protein necessary for development of midline glia and commissural axon pathways contains both EGF and LRR domains. Genes Dev. 1990;4:2169-87.

27. Plump AS, Erskine L, Sabatier C, Brose K, Epstein CJ, Goodman CS, et al. Slit1 and Slit2 cooperate to prevent premature midline crossing of retinal axons in the mouse visual system. Neuron. 2002;33:219-32.

28. Shu T, Sundaresan V, McCarthy MM, Richards LJ. Slit2 guides both precrossing and postcrossing callosal axons at the midline in vivo. J Neurosci. 2003;23:8176-84.

29. Hocking JC, Hehr CL, Bertolesi GE, Wu JY, McFarlane S. Distinct roles for Robo2 in the regulation of axon and dendrite growth by retinal ganglion cells. Mech Dev. 2010;127:36-48.

30. Lopez-Bendito G, Flames N, Ma L, Fouquet C, Di Meglio T, Chedotal A, et al. Robo 1 and Robo2 cooperate to control the guidance of major axonal tracts in the mammalian forebrain. J Neurosci. 2007;27:3395-407.

31. Burgess HA, Johnson SL, Granato M. Unidirectional startle responses and disrupted left-right co-ordination of motor behaviors in robo3 mutant zebrafish. Genes Brain Behav. 2009:8:500-11.

32. Shu T, Richards $\sqcup$. Cortical axon guidance by the glial wedge during the development of the corpus callosum. J Neurosci. 2001;21:2749-58.

33. Ten D. Anurans. In: Nieuwenhuys R, TenDonkelaar HJ, Nicholson C, editors, The central nervous system of vertebrates. 2nd ed. Heidelberg: Springer-Verlag; 1998. p. 1151-314.

34. van Mier P, ten Donkelaar HJ. Structural and functional properties of reticulospinal neurons in the early-swimming stage Xenopus embryo. J Neurosci. 1989;9:25-37.

35. van Mier P, Armstrong J, Roberts A. Development of early swimming in Xenopus laevis embryos: myotomal musculature, its innervation and activation. Neuroscience. 1989;32:113-26.

36. Nieuwkoop PD, Faber J. Normal Table of Xenopus laevis (Daudin). Amsterdam: North-Holland; 1967.

37. Moody SA, Kline MJ. Segregation of fate during cleavage of frog (Xenopus laevis) blastomeres. Anat Embryol (Berl). 1990;182:347-62.

38. Kawaguchi M, Sugahara Y, Watanabe T, Irie K, Ishida M, Kurokawa D, et al. Nervous system disruption and concomitant behavioral abnormality in early hatched pufferfish larvae exposed to heavy oil. Environ Sci Pollut Res Int. 2011;19:2488-97.

39. Takio Y, Kuraku S, Murakami Y, Pasqualetti M, Rijli FM, Narita Y, et al. Hox gene expression patterns in Lethenteron japonicum embryos-insights into the evolution of the vertebrate Hox code. Dev Biol. 2007;308:606-20.

40. Kuratani SC, Eichele G. Rhombomere transplantation repatterns the segmental organization of cranial nerves and reveals cell-autonomous expression of a homeodomain protein. Development. 1993;117:105-17.

41. Roberts A, Conte D, Hull M, Merrison-Hort R, al Azad AK, Buhl E, et al. Can simple rules control development of a pioneer vertebrate neuronal network generating behavior? J Neurosci. 2014;34:608-21. 
42. Geisen MJ, Di Meglio T, Pasqualetti M, Ducret S, Brunet JF, Chedotal A, et al. Hox paralog group 2 genes control the migration of mouse pontine neurons through slit-robo signaling. PLoS Biol. 2008;6:e142.

43. Nieuwenhuys R. Comparative anatomy of the cerebellum. Prog Brain Res. 1967;25:1-93.

44. Easter Jr SS, Taylor JS. The development of the Xenopus retinofugal pathway: optic fibers join a pre-existing tract. Development. 1989;107:553-73.

45. Key B, Anderson RB. Neuronal pathfinding during development of the rostral brain in Xenopus. Clin Exp Pharmacol Physiol. 1999;26:752-4.

46. Lázár G, Pál E. Neuronal connections through the posterior commissure in the frog Rana esculenta. J Hirnforsch. 1999;39:369-74.

47. Dickson BJ. Molecular mechanisms of axon guidance. Science. 2002:298:1959-64

48. Hutson LD, Chien CB. Pathfinding and error correction by retinal axons: the role of astray/robo2. Neuron. 2002;33:205-17.

49. Holmes G, Niswander L. Expression of slit-2 and slit-3 during chick development. Dev Dyn. 2001;222:301-7.

50. De Bellard ME, Rao Y, Bronner-Fraser M. Dual function of Slit2 in repulsion and enhanced migration of trunk, but not vagal, neural crest cells. J Cell Biol. 2003;162:269-79.

51. Farmer WT, Altick AL, Nural HF, Dugan JP, Kidd T, Charron F, et al. Pioneer longitudinal axons navigate using floor plate and Slit/Robo signals. Development. 2008;135:3643-53.

52. Mastick GS, Easter Jr SS. Initial organization of neurons and tracts in the embryonic mouse fore- and midbrain. Dev Biol. 1996;173:79-94.

53. Miyasaka N, Sato Y, Yeo SY, Hutson LD, Chien CB, Okamoto H, et al. Robo2 is required for establishment of a precise glomerular map in the zebrafish olfactory system. Development. 2005;132:1283-93.

54. Lee JS, Ray R, Chien CB. Cloning and expression of three zebrafish roundabout homologs suggest roles in axon guidance and cell migration. Dev Dyn. 2001:221:216-30.

55. Stanic K, Vera A, González M, Recabal A, Astuya A, Torrejón M, et al. Complementary expression of EphA7 and SCO-spondin during posteriorcommissure development. Front Neuroanat. 2014;8:49.

56. Bhidayasiri R, Plant GT, Leigh RJ. A hypothetical scheme for the brainstem control of vertical gaze. Neurology. 2000;54:1985-93.

57. Leichnetz GR, Gonzalo-Ruiz A, DeSalles AA, Hayes RL. The origin of brainstem afferents of the paramedian pontine reticular formation in the cat. Brain Res. 1987:422:389-97.

58. Ware M, Schubert FR. Development of the early axon scaffold in the rostral brain of the chick embryo. J Anat. 2011:219:203-16.

59. Schlosser G, Northcutt RG. Development of neurogenic placodes in Xenopus laevis. J Comp Neurol. 2000;418:121-46.

\section{Submit your next manuscript to BioMed Central and take full advantage of:}

- Convenient online submission

- Thorough peer review

- No space constraints or color figure charges

- Immediate publication on acceptance

- Inclusion in PubMed, CAS, Scopus and Google Scholar

- Research which is freely available for redistribution 